\title{
SCHULISCHER DAF-UNTERRICHT MIT UND OHNE ELEMENTE DER DRAMAPÄDAGOGIK: WORTSCHATZ IM FOKUS
}

Der Beitrag stellt eine Zusammenfassung der Dissertation der Autorin dar, die sich mit der Untersuchung der Unterschiede zweier Unterrichtsansätze hinsichtlich kognitiver und affektiver Variablen beschäftigt: dem herkömmlichen und dramapädagogischen DaF-Unterricht. Die Untersuchung wurde an zwei Primarschulen in Serbien mit 60 Fünftklässler/-innen durchgeführt, die während ihres ersten Lernjahrs anhand verschiedener Erhebungsinstrumente getestet und befragt wurden (s. Dragović 2019). Während in der Dissertation das Sprachenlernen im Allgemeinen und das Grammatiklernen im Einzelnen im Vordergrund stehen, wird der Fokus in diesem Beitrag auf das Vokabellernen verlagert. Am Beispiel einer Unterrichtseinheit wird demonstriert, worin die Unterschiede in der Wortschatzvermittlung im dramapädagogischen und herkömmlichen DaF-Unterricht bestehen. Aus den Erkenntnissen der Dissertation abgeleitete Handlungsempfehlungen für den Unterricht schließen den Beitrag ab.

Schlüsselwörter: Schulischer Kontext, Dramapädagogik, Fremdsprachenunterricht, DaF-Unterricht, Anfängerunterricht, Wortschatzvermittlung.

\section{Einführung}

Als DaF-Lehrerin, die mit der Grammatikvermittlung im eigenen Unterricht unzufrieden war, entschied die Autorin des vorlie-

georgina.dragovic@filum.kg.ac.rs 
genden Beitrags, eine Untersuchung durchzuführen, deren erstes Ziel darin bestand, der Frage nachzugehen, ob sich die Grammatikvermittlung durch den Einsatz der Dramapädagogik in zweierlei Hinsicht verbessern ließe: hinsichtlich des Grammatikerwerbs und der Einstellung zur Grammatik. Da jedoch viele Forschungsarbeiten vorliegen, die von positiven Effekten der Dramapädagogik berichten (vgl. etwa Tschurtschenthaler 2013; Jelic 2011; Even 2003), aber nur wenige, welche die Überprüfung der (hypothetisch) positiven Effekte intendieren (vgl. etwa Bora 2017; Kao 1994), wurde die Ausgangsmotivation schnell durch einen größeren Beweggrund erweitert: die Überprüfung der Effizienz der Dramapädagogik. ${ }^{1}$ Aus diesem Grund war es das Hauptanliegen, zu untersuchen, inwiefern sich der dramapädagogische und der herkömmliche DaF-Unterricht in einer Regelschule in Hinsicht auf kognitive (Lernleistung) und affektiv-motivationale Variablen (Motivation, Sprachangst und Einstellungen) unterscheiden (vgl. Dragović 2019: 199-119-122). Der bisher vorliegende Kenntnisstand erlaubte es, von der These auszugehen, dass «[d]ramapädagogische Verfahren [...] einen positiven Einfluss auf das Lernen und Lehren von Fremdsprachen im schulischen Unterricht haben [können]» (ebd.: 1).

\section{Warum dramapädagogisch?}

Die Dramapädagogik wird als performativer «Ansatz, der die Mittel des Theaters zu pädagogischen Zwecken einsetzt» (Tselikas 1999: 21) definiert. Da diese sehr weit gefasste Definition auch andere performative Ansätze umfassen kann, wird die Dramapädagogik an dieser Stelle näher verortet werden: Sie ist ein Ansatz, der durch den Einsatz performativer Mittel prozessorientiertes, interaktiv-kommunikatives, spielerisches, ganzheitliches, handlungsund erfahrungsbezogenes Sprachenlernen herbeiführt. ${ }^{2}$ Gerade in

1 Für eine Übersicht über die Forschungslage der Dramapädagogik s. Dragović (2019: 22-25, 103-114).

2 Für eine Abgrenzung der Dramapädagogik von anderen performativen Ansätzen s. Dragović (2019: 66-72). 
diesen Attributen liegen die Gründe für die Wahl des Unterrichtsansatzes für die Untersuchung. Grundsätzlich sollte es mit der Dramapädagogik möglich sein, entsprechende Nachteile des heutigen Fremdsprachenunterrichts - wie z. B. lehrwerkzentrierter, bewegungsarmer, emotional wenig ansprechender, stark kognitiv ausgerichteter Unterricht und Einsatz herkömmlicher Verfahren zu überbrücken. ${ }^{3}$ Die Idee dahinter ist, durch den Einsatz der Dramapädagogik motorischen, kreativen, ästhetischen, emotionalen und empathischen Faktoren mehr Gewicht zu verleihen (vgl. Even 2003: 21), weil davon ausgegangen wird, dass dann die Lern- und Behaltensleistung, Motivation, Einstellungen und Sprachangst im Fremdsprachenunterricht optimiert werden können.

\section{Also nur dramapädagogisch?}

Soll der dramapädagogische Fremdsprachenunterricht das Lehrwerk, das Sitzen in Bänken, neutrale Inhalte sowie „reine“ Kopfarbeit ersetzen? Keineswegs; vielmehr ginge es darum, den herkömmlichen Fremdsprachenunterricht beizubehalten, aber durch die Elemente der Dramapädagogik zu bereichern, damit nicht nur die Mängel des herkömmlichen, sondern auch jene des dramapädagogischen Fremdsprachenunterrichts beseitigt werden, nämlich das Problem der Integration des dramapädagogischen Ansatzes in den schulischen Kontext (vgl. Jelic 2011: 133). Indem also der „normale“ bzw. ein lehrplangestützter, lehrwerkzentrierter und zeitlich begrenzter Fremdsprachenunterricht als Basis genommen wird, sollen die Grenzen, innerhalb derer sich die Dramapädagogik bewegen darf, gesetzt werden. Dementsprechend werden für den Fremdsprachenunterricht nur diejenigen dramapädagogischen Aktivitäten und Verfahren gewählt, die kursorisch eingesetzt werden können, um die vorgegebenen Lernziele, die im schulischen Kontext erreicht werden sollen, verfolgen zu können (vgl. Dragović 2019: 127-131).

3 Eine extensive Auseinandersetzung mit den Nachteilen des herkömmlichen Fremdsprachenunterrichts findet sich in Dragović (2019: 12-21, 61-63) 


\section{Doch herkömmlich?}

Kritisiert werden könnte auf beiden Fronten: Aufseiten des herkömmlichen Unterrichts könnte gegen den Einbau performativer Elemente wegen unzureichender empirischer Evidenz argumentiert werden. Aufseiten des dramapädagogischen Unterrichts könnten Argumente gegen den kursorischen Ablauf vorgebracht werden, weil vermutet wird, dass das ganze Potenzial der Dramapädagogik ausgeschöpft werden kann, wenn dem Unterricht keine Grenzen gesetzt werden, $d$. h. wenn nichts vorgegeben und antizipierbar ist.

M. E. erweisen sich sowohl der herkömmliche als auch der dramapädagogische Fremdsprachenunterricht als schlechte Lösungsoptionen für den schulischen Kontext. Der herkömmliche aus den bereits genannten Gründen; der dramapädagogische wegen der Unvorhersehbarkeit des Ablaufs des dramapädagogischen Prozesses. Deshalb wäre die Zusammenführung der beiden Ansätze einen Versuch wert -, auch wenn dabei in Kauf genommen werden muss, dass potenziell gute Effekte, die der Dramapädagogik zugeschrieben werden, nicht auftreten könnten. ${ }^{4}$

\section{Lehrwerkorientierter DaF-Unterricht mit und ohne dramapädagogische Elemente im Vergleich}

Da weder der eine noch der andere Unterrichtsansatz dem schulischen Fremdsprachenunterricht gänzlich gerecht werden können, wurden für die Untersuchung zwei Unterrichtskonzepte entwickelt: eins, das dramapädagogische Elemente in den lehrwerkorientierten DaF-Unterricht miteinbezieht, und ein weiteres, das dramapädagogische Elemente aus dem lehrwerkorientierten DaF-Unterricht ausklammert bzw. herkömmliche Verfahren einschließt (vgl. ebd.). D. h., das Lehrwerk bildet die Basis beider Unterrichtskonzepte. Der Unterschied liegt lediglich darin, wie die Lehrwerkinhalte im Unterricht behandelt werden. Da in der Disser-

4 Für eine Übersicht über die Pro- und Contra-Argumente s. Dragović (2019: 8-9, 114-118, 253-254). 
tation aufgezeigt wird, wie der erste Sprachkontakt und die Grammatikvermittlung in einer „normalen“ und dramaorientierten Stunde ablaufen kann (vgl. ebd.: 131-138), soll im Folgenden aufgezeigt werden, inwiefern die Herangehensweisen an Wortschatzvermittlung unterschiedlich sind. Die Unterrichtseinheit richtet sich an dieselbe Zielgruppe wie in der Untersuchung: Schüler/-innen (SuS) im Anfangsunterricht in DaF an serbischen Grundschulen.

\section{Unterrichtseinheit „Schulfächer“}

Wie werden Vokabeln und Chunks normalerweise in einem klassischen, lehrwerkzentrierten DaF-Unterricht in Serbien eingeführt und eingeübt? Darüber unterhielt sich die Autorin mit zwei Fachkolleginnen bei der Erstellung des Kontrollkonzepts, das den schulischen Alltag in Serbien darstellen sollte. Aus der Diskussion resultierte, dass das Übersetzen von Wörtern und festen Wendungen aus Lese- und Hörtexten oder das Einführen mithilfe von Bildern und Fotos zu den häufigsten Semantisierungstechniken zählen. Systematisiert werden die neuen Wörter, indem die Lehrperson (LP) die Wörter je nach Geschlecht in drei Spalten an die Tafel schreibt und den SuS dazu rät, diese mit Farben zu markieren (grün, rot und blau). Etwas seltener wird der Wortschatz mittels Definitionen, Synonymen/Antonymen, Übersetzung in der ersten Fremdsprache bzw. Englisch oder Mnemotechniken eingeführt. Noch seltener werden Wörter in den Stunden so eingeübt, dass die LP eine Rückmeldung darüber bekommt, ob die SuS die ausgesprochenen Wörter gleichzeitig verstehen. Meistens ist es der Fall, dass z. B. Dialoge durch das Einsetzen von neuen Wörtern oder Chunks variiert werden, ohne auf die Bedeutung einzugehen. Die Bedeutung spielt meistens nur dann eine Rolle, wenn man Aussagen über sich oder jemanden aus der Klasse machen soll. Je nachdem, wie man den Wortschatz einübt, werden verschiedene Sozialformen eingesetzt: Einzelarbeit (schriftliches Lösen von Wortschatzübungen), Partner - oder Gruppenarbeit (gegenseitiges Abfragen) oder Arbeit im Plenum (Kettenübung mit Frage-Antwort-Muster). Für 
spielerische Aktivitäten, wie das Gedächtnisspiel, das Quartett u. Ä., geschweige denn dramapädagogische Aktivitäten, wird fast nie Zeit eingeräumt.

Angelehnt an die Diskussionsergebnisse wurde das Kontrollkonzept erstellt, dass dem DaF-Unterricht in der Kontrollgruppe diente, der in der Dissertation als herkömmlicher DaF-Unterricht bezeichnet wird. In Tabelle 1 kann eingesehen werden, wie das Wortfeld Schulfächer in der 12. Deutschstunde in der Kontrollgruppe eingeführt und geübt wurden. Als Grundlage für den Unterricht diente das Lehrwerk Prima A1 (Jin et al. 2012).

\begin{tabular}{|l|l|l|l|l|}
\hline $\begin{array}{l}\text { Stunde: } \\
\text { Thema: } \\
\text { Klasse: } \\
\text { Lernziel: }\end{array}$ & $\begin{array}{l}\text { 12. Stunde } \\
\text { Schulfächer } \\
\text { Kontrollgruppe } \\
\text { (5. Klassenstufe) } \\
\text { Die SuS können Schulfächer } \\
\text { benennen sagen, welche sie } \\
\text { (nicht) mögen. }\end{array}$ & & \\
\hline $\begin{array}{l}\text { Unterrichtsschritte / } \\
\text { Lehr- und Lernaktiv- } \\
\text { itäten }\end{array}$ & $\begin{array}{l}\text { Metho- } \\
\text { disches } \\
\text { Handeln }\end{array}$ & $\begin{array}{l}\text { Sozialformen } \\
\text { und Lehr- } \\
\text { methoden }\end{array}$ & $\begin{array}{l}\text { Hilfsmit- } \\
\text { tel und } \\
\text { Material }\end{array}$ & Zeit \\
\hline $\begin{array}{l}\text { Die LP schreibt „Meine } \\
\text { Klasse“ an die Tafel } \\
\text { und fragt die SuS, ob } \\
\text { sie wüssten, was die } \\
\text { Überschrift bedeutet. } \\
\text { Dann bittet sie die SuS, } \\
\text { das Buch auf S. 13 zu } \\
\text { öffnen. Die SuS sagen } \\
\text { dann auf Serbisch, was } \\
\text { "Meine Klasse“ bedeu- } \\
\text { tet, falls sie es nicht } \\
\text { früher erraten haben. }\end{array}$ & $\begin{array}{l}\text { Einführung } \\
\text { ins Thema }\end{array}$ & $\begin{array}{l}\text { Arbeit im Ple- } \\
\text { num / Gezielte } \\
\text { Lehrerfragen. }\end{array}$ & $\begin{array}{l}\text { Kursbuch. } \\
\text { Tafel }\end{array}$ & 3 Min. \\
& & & \\
& & & \\
\end{tabular}




\begin{tabular}{|c|c|c|c|c|}
\hline $\begin{array}{l}\text { Die SuS hören den Di- } \\
\text { alog (S. 14, CD 12). Da- } \\
\text { nach fragt die LP, was } \\
\text { sie verstanden hätten. } \\
\text { Danach schlagen die } \\
\text { SuS das Buch auf S. } 14 \\
\text { auf, hören den Dialog } \\
\text { noch einmal und lesen } \\
\text { mit. }\end{array}$ & $\begin{array}{l}\text { Hörver- } \\
\text { stehen. } \\
\text { Vorlesen }\end{array}$ & $\begin{array}{l}\text { Einzelarbeit } \\
\text { / Gezielte } \\
\text { Lehrerfragen. } \\
\text { Hörübung. } \\
\text { Vorlesen }\end{array}$ & $\begin{array}{l}\text { Kursbuch. } \\
\text { Tafel. CD. } \\
\text { CD-Player }\end{array}$ & 4 Min. \\
\hline $\begin{array}{l}\text { Die LP fragt die S, was } \\
\text { das Thema des Dialogs } \\
\text { sei. Danach schreibt } \\
\text { sie alle Schulfächer auf } \\
\text { Serbisch an die Tafel } \\
\text { und erteilt den SuS die } \\
\text { Aufgabe, die Fächer } \\
\text { mit Hilfe des Buchs zu } \\
\text { übersetzen. }\end{array}$ & $\begin{array}{l}\text { Erarbeitung } \\
\text { eines Wort- } \\
\text { feldes }\end{array}$ & $\begin{array}{l}\text { Arbeit im Ple- } \\
\text { num / Gezielte } \\
\text { Lehrerfragen. } \\
\text { Textüber- } \\
\text { setzung }\end{array}$ & $\begin{array}{l}\text { Tafel. } \\
\text { Kursbuch }\end{array}$ & 3 Min. \\
\hline $\begin{array}{l}\text { Die LP schreibt die } \\
\text { Fächer auf Deutsch } \\
\text { an die Tafel: Deutsch, } \\
\text { Englisch, Serbisch, } \\
\text { Bio (Biologie), Geo } \\
\text { (Geografie), Mathe } \\
\text { (Mathematik), Ge- } \\
\text { schichte, Sport, Reli- } \\
\text { gion, Zeichnen, Musik. }\end{array}$ & $\begin{array}{l}\text { Arbeit } \\
\text { auf dem } \\
\text { Wortfeld } \\
\text { "Schul- } \\
\text { fächer" }\end{array}$ & $\begin{array}{l}\text { Frontal / } \\
\text { Gemeinsame } \\
\text { Wissenserar- } \\
\text { beitung. } \\
\text { Lehrervortrag. }\end{array}$ & Tafel & 5 Min. \\
\hline $\begin{array}{l}\text { Kettenübung: Die LP } \\
\text { beginnt mit der Frage: } \\
\text { „Was magst du?“ und } \\
\text { der/die Schüler/in } \\
\text { antwortet z. B. „Ich } \\
\text { mag Bio.“ Dann fragt } \\
\text { der/die Schüler/in } \\
\text { den/die Nächste/n. } \\
\text { Die SuS fragen ein- } \\
\text { ander der Reihe nach, } \\
\text { bis die ganze Klasse } \\
\text { gefragt und geantwor- } \\
\text { tet hat bzw. bis die LP } \\
\text { gefragt wird. }\end{array}$ & $\begin{array}{l}\text { Wortschatz } \\
\text { üben }\end{array}$ & $\begin{array}{l}\text { Frontalimpuls. } \\
\text { Interaktion } \\
\text { mit wechseln- } \\
\text { dem Partner } \\
\text { im Plenum / } \\
\text { Kettenübung. } \\
\text { Fragen und } \\
\text { Antworten }\end{array}$ & Tafel & 15 Min. \\
\hline
\end{tabular}


Georgina V. Dragović

\begin{tabular}{|c|c|c|c|c|}
\hline $\begin{array}{l}\text { Freiwillige melden } \\
\text { sich, um den Dialog } \\
\text { auf S. } 14 \text { vorzulesen. } \\
\text { Die anderen hören zu } \\
\text { und korrigieren die } \\
\text { Aussprache. }\end{array}$ & $\begin{array}{l}\text { Aussprache } \\
\text { üben. } \\
\text { Aufmerk- } \\
\text { samkeit } \\
\text { erhöhen }\end{array}$ & $\begin{array}{l}\text { Gruppenarbeit } \\
\text { / Beobachter- } \\
\text { auftrag. Vor- } \\
\text { lesen. Schüler- } \\
\text { korrektur }\end{array}$ & Kursbuch & 6 Min. \\
\hline $\begin{array}{l}\text { Die LP fordert die } \\
\text { SuS auf, den Dialog zu } \\
\text { variieren. Sie sollen } \\
\text { einen neuen Dialog ins } \\
\text { Heft schreiben und ihn } \\
\text { in der nächsten Stunde } \\
\text { zu zweit vortragen. }\end{array}$ & $\begin{array}{l}\text { Schreiben } \\
\text { üben }\end{array}$ & $\begin{array}{l}\text { Partnerarbeit / } \\
\text { Schreiben }\end{array}$ & & $\begin{array}{l}9 \\
\text { Min. }\end{array}$ \\
\hline $\begin{array}{l}\text { Für diejenigen SuS, die } \\
\text { schnell sind, gilt fol- } \\
\text { gende Aufgabe bis zum } \\
\text { Ende der Stunde: } \\
\text { Die SuS fragen ein- } \\
\text { ander ab, indem sie } \\
\text { die folgende Frage } \\
\text { stellen: „Wie heißt ... } \\
\text { auf Deutsch?“ }\end{array}$ & $\begin{array}{l}\text { Wortschatz } \\
\text { einüben. }\end{array}$ & $\begin{array}{l}\text { Partnerarbeit } \\
\text { / Fragen und } \\
\text { Antworten. }\end{array}$ & & \\
\hline
\end{tabular}

Tabelle 1: 12. Unterrichtseinheit aus dem Kontrollkonzept

Im dramapädagogischen DaF-Unterricht wird der Wortschatz normalerweise mit dem Körper erarbeitet. Damit ist nicht nur die pantomimische Darstellung gemeint. Die Vielfalt an Möglichkeiten reicht von Standbildern über das Zeichnen von imaginären Objekten mit dem Körper bis zum Geräuschmachen - sei es mit der Stimme oder mit Objekten. Die LP greift zu den herkömmlichen Semantisierungstechniken nur im Falle, dass die SuS Verstehenshilfen benötigen. Neben den zahlreichen Techniken gibt es eine, die sich wunderbar für die Einführung in ein neues Thema eignet und außerdem für die Dramapädagogik ausschlaggebend ist. Das ist die sog. Lehrer-in-der-Rolle-Technik (LiRo-Technik, org. Teacher in Role). ${ }^{5}$ Wie der Name sagt, besteht der Sinn des Verfahrens darin,

5 Ausschlaggebend ist die LiRo-Technik, weil sie von Dorothy Heathcote, einer der Pionir/-innen der britischen Dramapädagogik, entwickelt wurde (vgl. Bolton 1998). 
dass die LP aus einer Rolle agiert und die SuS in die dramatische Welt miteinzieht (s. Tabelle 2). ${ }^{6}$ Eingeübt werden können die Wörter ebenfalls mit Bewegung oder durch körperintegriertes Spielen. Bspw. können Wörter vor der Klasse pantomimisch dargestellt oder als Standbild verkörpert werden. Gleichfalls können Wörter und Wendungen geübt werden, indem sie rhythmisch mit Bewegung nachgesprochen sowie mit dem Finger auf den Rücken oder in die Luft geschrieben werden. Wörter können auch mit realen Objekten eingeübt werden, indem sie bspw. unter einem Tischtuch zum Anfassen versteckt werden oder indem man sie den SuS mit verbundenen Augen zum Riechen reicht. Um sie in den Kontext einordnen zu können, werden im Nachhinein Rollenspiele entwickelt, die je nach Sprachstand ebenfalls auf verschiedene Weisen inszeniert werden können. Neue Wörter müssen aber nicht zwingend in eine Inszenierung einer kommunikativen Handlungssituation münden; es können auch kreative Wortbildgestaltungen, Reime, Gedichte oder Geschichten erfunden werden.

Die Palette ist im dramapädagogischen Fremdsprachenunterricht breit gefächert, weshalb es schwierig ist, alle Möglichkeiten aufzuzeigen. Aus diesem Grund sei auch an dieser Stelle exemplarisch auf die 12. Deutschstunde in der Interventionsgruppe verwiesen (s. Tabelle 2). Die nachstehende Unterrichtsvorbereitung bezieht sich wie die vorige auf das Lehrwerk Prima A1 (Jin et al. 2012).

\begin{tabular}{|c|c|c|c|c|}
\hline $\begin{array}{l}\text { Stunde: } \\
\text { Thema: } \\
\text { Klasse: } \\
\text { Lernziel: }\end{array}$ & \multicolumn{4}{|c|}{$\begin{array}{l}\text { 12. Stunde } \\
\text { Schulfächer } \\
\text { Experimentalklasse (5. Klassenstufe) } \\
\text { Die S können Schulfächer benennen und } \\
\text { sagen, welche sie (nicht) mögen. }\end{array}$} \\
\hline $\begin{array}{l}\text { Unterrichtsschritte / } \\
\text { Lehr- und } \\
\text { Lernaktivitäten }\end{array}$ & $\begin{array}{l}\text { Methodisches } \\
\text { Handeln }\end{array}$ & $\begin{array}{l}\text { Sozialformen } \\
\text { und } \\
\text { Lehrmethoden }\end{array}$ & $\begin{array}{l}\text { Hilfsmittel } \\
\text { und } \\
\text { Material }\end{array}$ & Zeit \\
\hline
\end{tabular}

6 Für eine genauere Beschreibung dieser Inszenierungstechnik s. Schewe (2000: 96). 
Die LP tritt als ein/e verkleidete/r Schüler/in in die Klasse, begrüßt alle und stellt sich als der/ die Neue mit verstellter Stimme vor (Angaben auf S. 14). Danach setzt sich die LP bzw. der/ die neue Schüler/in neben eine/n Schüler/ in, holt die Schulsachen aus dem Rucksack hervor und fragt: „Was haben wir jetzt? Mathe? Bio? Deutsch?" Die SuS lachen, sind erstaunt und warten darauf, was als Nächstes passiert. Die LP wiederholt die Fragen, bis ein/e Schüler/in „Deutsch“ sagt. Danach erwidert die LP: „Deutsch? Ich mag Deutsch. Deutsch ist super. Magst du Deutsch? Und du? Und ihr? Magst du Mathe?" Die SuS antworten mit einem Kopfschütteln / Nicken (im besten Fall mit „Ja/ Nein."). Dann springt die LP auf und sagt: „Oops, ich muss dringend auf die Toilette. Wo ist denn das WC?" Die SuS weisen ihr den Weg und die LP rennt raus. Nach einigen Sekunden kommt die LP als sie selbst ins Klassenzimmer zurück und wartet auf die Reaktion der SuS. Sie reden darüber, was gerade passiert ist.
Aufmerksamkeit wecken.

Entfachung von Neugier. Einführung ins Thema. Wiederholung
Arbeit im Plenum

/ LiRo- und LaRo-Technik. Gezielte Lehrerfragen. Schülergespräch
Rucksack. Brille. Heft. Bleistift
5

Min. 


\begin{tabular}{|c|c|c|c|c|}
\hline $\begin{array}{l}\text { Alle sitzen in einem } \\
\text { Kreis. Die LP wirft } \\
\text { einem/r Schüler/in } \\
\text { einen imaginären Ball } \\
\text { zu und fordert ihn/ } \\
\text { sie auf, ein Schulfach } \\
\text { auf Serbisch zu sagen. } \\
\text { Dann fragt die LP, ob } \\
\text { sich jemand daran } \\
\text { erinnern könne, wie } \\
\text { das Fach auf Deutsch } \\
\text { heißt und schreibt es } \\
\text { an die Tafel. An- } \\
\text { schließen sagen es } \\
\text { alle nach (zuerst laut, } \\
\text { dann leise, traurig,...). } \\
\text { Dann wirft der/die } \\
\text { Schüler/in den Ball } \\
\text { weiter und das Ganze } \\
\text { wird wiederholt, bis } \\
\text { alle Fächer an der Tafel } \\
\text { sind: Deutsch, Englisch, } \\
\text { Serbisch, Bio (Biologie), } \\
\text { Geo (Geografie), Mathe } \\
\text { (Mathematik), Ges- } \\
\text { chichte, Sport, Religion, } \\
\text { Zeichnen, Musik. }\end{array}$ & $\begin{array}{l}\text { Themati- } \\
\text { sierung. } \\
\text { Arbeit auf } \\
\text { dem Wortfeld } \\
\text { „Schulfächer“. } \\
\text { Aktivierung } \\
\text { der SuS. } \\
\text { Aufwärmen } \\
\text { der Stimme als } \\
\text { Vorbereitung } \\
\text { für die } \\
\text { Bühnenarbeit. }\end{array}$ & $\begin{array}{l}\text { Frontalimpuls. } \\
\text { Großgruppe } \\
\text { im Sitzkreis / } \\
\text { Lernspiel. Cho- } \\
\text { risches Sprechen. } \\
\text { Erproben der } \\
\text { Stimme }\end{array}$ & Ball, Tafel & $\begin{array}{l}7 \\
\text { Min. }\end{array}$ \\
\hline
\end{tabular}




\begin{tabular}{|c|c|c|c|}
\hline $\begin{array}{l}\text { Jedem/r Schüler/ } \\
\text { in wird eines der } \\
\text { Fächer zugeteilt. Ein/e } \\
\text { freiwillige/r Schüler/ } \\
\text { in stellt sich in die } \\
\text { Mitte und sein/ihr } \\
\text { Stuhl wird aus dem } \\
\text { Kreis genommen. Nun } \\
\text { sagt er/sie z. B. „Bio“. } \\
\text { Diejenigen, denen } \\
\text { dieses Wort zugeteilt } \\
\text { wurde, müssen nun } \\
\text { versuchen ihre Plätze } \\
\text { zu wechseln, während } \\
\text { der/die Schüler/in in } \\
\text { der Mitte versucht, } \\
\text { einen Platz zu ergat- } \\
\text { tern. Wer „platzlos“ } \\
\text { bleibt, kommt in } \\
\text { die Mitte und macht } \\
\text { weiter. Wenn der/die } \\
\text { Schüler/in in der Mitte } \\
\text { "Schulfach“ sagt, müs- } \\
\text { sen alle einen neuen } \\
\text { Platz suchen. }\end{array}$ & $\begin{array}{l}\text { Wortschatzfes- } \\
\text { tigung. } \\
\text { Förderung } \\
\text { der Reak- } \\
\text { tionsfähigkeit. } \\
\text { Förderung von } \\
\text { Raum- und } \\
\text { Bewegungs- } \\
\text { gefühl. }\end{array}$ & $\begin{array}{l}\text { Großgruppe } \\
\text { im Sitzkreis / } \\
\text { Lernspiel. }\end{array}$ & $\begin{array}{l}3 \\
\text { Min. }\end{array}$ \\
\hline $\begin{array}{l}\text { Die LP bittet den/ } \\
\text { die Schüler/in in der } \\
\text { Mitte ihr die Frage } \\
\text { „Was magst du?“ zu } \\
\text { stellen. Die LP antwor- } \\
\text { tet: „Ich mag Sport.“ } \\
\text { Dann erklärt sie, dass } \\
\text { nun alle, auf deren } \\
\text { Kärtchen „Sport“ steht, } \\
\text { „Ich auch.“ sagen und } \\
\text { die Plätze tauschen } \\
\text { müssen. Wer als } \\
\text { Nächster in der Mitte } \\
\text { steht, stellt jemandem } \\
\text { im Kreis die nächste } \\
\text { Frage. }\end{array}$ & $\begin{array}{l}\text { Wortschatzfes- } \\
\text { tigung. } \\
\text { Grammatik- } \\
\text { wiederholung. } \\
\text { Förderung } \\
\text { der Reak- } \\
\text { tionsfähigkeit. } \\
\text { Förderung von } \\
\text { Raum- und } \\
\text { Bewegungs- } \\
\text { gefühl. }\end{array}$ & $\begin{array}{l}\text { Variation von } \\
\text { Primärimpuls. } \\
\text { Interaktion mit } \\
\text { wechselndem } \\
\text { Partner im Sitz- } \\
\text { kreis / Lernspiel }\end{array}$ & $\begin{array}{l}7 \\
\text { Min. }\end{array}$ \\
\hline
\end{tabular}




\begin{tabular}{|c|c|c|c|c|}
\hline $\begin{array}{l}\text { Nun erklärt die LP, } \\
\text { dass nun alle, die } \\
\text { das in der Antwort } \\
\text { erwähnte Schulfach } \\
\text { nicht auf dem Kärtch- } \\
\text { en haben, „Ich nicht.“ } \\
\text { sagen und die Plätze } \\
\text { tauschen müssen. }\end{array}$ & $\begin{array}{l}\text { Wortschatzfes- } \\
\text { tigung. } \\
\text { Grammatik- } \\
\text { wiederholung. } \\
\text { Förderung } \\
\text { der Reak- } \\
\text { tionsfähigkeit. } \\
\text { Förderung von } \\
\text { Raum- und } \\
\text { Bewegungs- } \\
\text { gefühl. }\end{array}$ & $\begin{array}{l}\text { Variation von } \\
\text { Primärimpuls. } \\
\text { Interaktion mit } \\
\text { wechselndem } \\
\text { Partner im Sitz- } \\
\text { kreis / Lernspiel }\end{array}$ & & $\begin{array}{l}7 \\
\text { Min. }\end{array}$ \\
\hline $\begin{array}{l}\text { Die LP bittet die SuS } \\
\text { zu zweit ein Fach zu } \\
\text { wählen und es als } \\
\text { Standbild darzus- } \\
\text { tellen. Freiwillige } \\
\text { Paare präsentieren } \\
\text { ihre Standbilder } \\
\text { und die anderen SuS } \\
\text { raten, welches Fach } \\
\text { dargestellt ist. Die LP } \\
\text { macht Fotos von den } \\
\text { SuS (die Fotos bringt } \\
\text { sie nächste Stunde } \\
\text { mit, damit sie die SuS } \\
\text { ausschneiden und den } \\
\text { Fächern zuordnen } \\
\text { können). }\end{array}$ & $\begin{array}{l}\text { Aufwärmen } \\
\text { und Sensibili- } \\
\text { sierung für } \\
\text { die Dramatis- } \\
\text { ierung. SuS } \\
\text { interpretieren } \\
\text { nonverbalen } \\
\text { Ausdruck } \\
\text { und üben den } \\
\text { Wortschatz ein. }\end{array}$ & $\begin{array}{l}\text { Frontalimpuls, } \\
\text { Partnerarbeit / } \\
\text { Standbild. Beo- } \\
\text { bachterauftrag. }\end{array}$ & Fotoapparat & $\begin{array}{l}11 \\
\text { Min. }\end{array}$ \\
\hline $\begin{array}{l}\text { Die SuS bekommen die } \\
\text { Fächer auf einem Blatt } \\
\text { Papier zum Ausschnei- } \\
\text { den und müssen sie } \\
\text { für die nächste Stunde } \\
\text { ins Heft kleben, eine } \\
\text { passende Zeichnung } \\
\text { dazu malen und Platz } \\
\text { für Fotos (Standbilder) } \\
\text { übrig lassen. }\end{array}$ & & $\begin{array}{l}\text { Frontal / } \\
\text { Lehrervortrag }\end{array}$ & Kopien & $\begin{array}{l}5 \\
\text { Min. }\end{array}$ \\
\hline
\end{tabular}

Tabelle 2: 12. Unterrichtseinheit aus dem Interventionskonzept

Werden die Unterrichtsvorbereitungen miteinander verglichen, dann sticht hervor, dass im herkömmlichen DaF-Unterricht nicht 
mit dem Körper gearbeitet wird, dass das unterrichtliche Geschehen hauptsächlich durch die LP gelenkt wird, dass mehr schriftlich und im Sitzen gearbeitet wird und dass die formale, aber keine inhaltliche Wissenssicherung gewährleistet wird. Im dramapädagogischen DaF-Unterricht indessen wird sehr viel Wert auf das Gefühlvolle, Stimmliche, Imaginäre und Körperliche gelegt. Ergo wird ganzheitlich gelehrt und gelernt.

Wie bereits erwähnt, stehen sich im dramapädagogischen Diskurs Befürworter/-innen und Skeptiker/-innen gegenüber. In Anbetracht der empirischen Grundlage ist dies durchaus legitim. Um diese Forschungslücke für den Anfängerunterricht im schulischen Kontext für den Bereich DaF schließen zu können, wurde eine Untersuchung durchgeführt, die der Überprüfung sämtlicher zugeschriebener bzw. bezweifelter Effekte der Dramapädagogik nachgeht. Weitere Hintergrundinformationen zur Studie sowie deren Befunde werden im folgenden Abschnitt zusammengefasst.

\section{Untersuchung und Ergebnisse}

Um zu untersuchen, ob es Unterschiede zwischen den besagten Unterrichtsansätzen gibt, wurde eine Interventionsstudie an einer serbischen Primarschule durchgeführt (vgl. Dragović 2019). Befragt und getestet wurden $60 \mathrm{SuS}$ der 5. Klassenstufe aus drei Klassen im Zeitraum von einem Schuljahr. Neben dem Alter war ihnen allen Folgendes gemeinsam: Serbisch als Muttersprache, Englisch als erste Fremdsprache, keine Vorkenntnisse in Deutsch, ähnliches soziales Milieu, gleiche Lerntradition und -erfahrung sowie vergleichbare Geschlechts- und Leistungsverteilung. Verglichen wurde eine Klasse, die dramapädagogisch unterrichtet wurde (Interventionsgruppe, $n=18$ ), mit zwei anderen, die herkömmlich unterrichtet wurden (Kontrollgruppe, $n=42$ ). Es wurden drei Befragungsinstrumente eingesetzt: eine Batterie zur Motivationserfassung, ein Fragebogen zur Erfassung der Einstellungen zum Grammatiklernen und eine Gruppendiskussion zur Evaluation des dramapädagogischen DaF-Unterrichts. Weiterhin wurden drei Testtypen einge- 
setzt: viermal vier verschiedene Lernfortschrittstests, zweimal ein C-Test in Deutsch und einmal ein C-Test in Englisch. Die gewonnenen Daten wurden qualitativ und quantitativ ausgewertet. Keiner der Fälle wurde aus den Analysen ausgeschlossen.?

Wie die Ergebnisse dieser Studie zeigen, sind signifikante, aber auch nicht signifikante Unterschiede zwischen den verglichenen Untersuchungsgruppen vorhanden. Zu den eindeutig signifikanten Unterschieden zählen die Unterschiede im Hinblick auf den Lernfortschritt, den allgemeinen Sprachstand, die Rechtschreibung, die mündliche Produktion, die Aussprache, die Motivation, die Einstellungen zur Lernsituation und die affektive Einstellung zum Grammatiklernen. Dabei konnten in der dramapädagogisch unterrichteten Klasse höhere Werte für all diese Variablen verzeichnet werden. Im Gegensatz dazu gibt es keine Unterschiede in Hinsicht auf die Behaltensleistung, das Interesse am Fremdsprachenlernen, die Sprachangst, das Verstehen grammatischer Strukturen und die kognitive Einstellung zum Grammatiklernen. Als positiv betrachtet werden kann die Tatsache, dass die Werte dieser Variablen sowohl in den herkömmlich als auch in den dramapädagogisch unterrichteten Klassen deskriptiv betrachtet gut ausfallen. Während die besagten Ergebnisse leicht interpretiert werden können, gibt es jene, welche mehrdeutig ausfallen. Dies trifft auf den Befund zur schriftlichen Produktion, weil er grenzsignifikant ausfällt, und auf den Befund zur Grammatikkompetenz, weil sich signifikante und nicht signifikante Ergebnisse gegenüberstehen. Weiterhin gibt es qualitativ ermittelte Ergebnisse, die mit den quantitativ gewonnenen nicht konform gehen - wie z. B. die Befunde zur Angst, Behaltensleistung, Grammatikkompetenz und zum Verstehen grammatischer Strukturen. Zudem liefern die qualitativ ermittelten Ergebnisse Hinweise darauf, dass die Konzentrationsleistung und gewisse überfachliche Kompetenzen gefördert werden können. Nicht zuletzt gibt es quantitativ ermittelte Ergebnisse, die als interessante Nebenprodukte gesehen werden können, weil sie sich auf keine der aufgestellten

$7 \quad$ Ausführliche Informationen zu Stichprobe, Untersuchungsdesign, -verlauf und -instrumente sowie Datenaufbereitung und -auswertung finden sich im vierten Kapitel der Arbeit (s. Dragović 2019: 119-165). 
Hypothesen beziehen. So konnte bspw. festgestellt werden, dass die Einstellungen zum Grammatiklernen konsistent sind und dass die motivational-affektiven Konstrukte und die Einstellungskomponenten der Grammatik nicht miteinander korrelieren. ${ }^{8}$

\section{Bilanz und Fazit}

Ob sich die Dramapädagogik positiv auf die Wortschatzvermittlung auswirkte, kann anhand der Befundlage nicht gesagt werden. Dennoch erlaubt die Tatsache, dass die dramapädagogisch unterrichteten SuS in Hinsicht auf den Lernfortschritt und den Sprachstand signifikant besser als ihre Peers in der Kontrollgruppe waren, die Annahme, dass die Wahrscheinlichkeit, dass die Dramapädagogik den Wortschatzerwerb begünstigte, sehr groß ist. Ob nun die Dramapädagogik bei der Wortschatzvermittlung vorzuziehen wäre, hängt $m$. E. davon ab, wie sie sich auf den Spracherwerb im Gesamten auswirkte. Betrachtet man die Resultate dieser Studie holistisch, kann daraus geschlossen werden, dass sich die Ausgangsthese der Autorin bestätigt hat: „Dramapädagogische Verfahren können einen positiven Einfluss auf das Lernen und Lehren von Fremdsprachen im schulischen Unterricht haben" (vgl. ebd.: 262). Gewiss können nicht alle Fertigkeiten und Aspekte des DaF-Unterrichts mithilfe der Dramapädagogik optimiert werden. Fakt ist jedoch, dass sich die Dramapädagogik auf keine der gemessenen Variablen negativ auswirkte. Deswegen sei an dieser Stelle noch einmal dazu geraten, den DaF-Unterricht gelegentlich dramapädagogisch zu gestalten. Zumindest kann in der Arbeit mit einer Zielgruppe wie dieser hier, d. h. mit Kindern im schulischen DaF-Unterricht vorausgesetzt werden, dass die Dramapädagogik lern- und motivationsförderlich auf die SuS wirken dürfte.

8 Die Ergebnisse der Untersuchung können im vierten, die dazugehörigen Diskussionen im fünften Kapitel der Arbeit nachgesehen werden (vgl. Dragović 2019: 166-249). 


\section{LITERATURVERZEICHNIS}

Bolton 1998: Bolton G. Acting in Classroom Drama - critical analysis. Stokeon-Trent, United Kingdom: Trentham.

Bora 2017: Bora S. F. Beyond the Mere Word. Exploring the Language of Drama through Text- and Performance-based Approaches for Developing L2 Oral Skills, [http://repository.essex.ac.uk/], (Zugriff am 10.10.2019).

Dragović 2019: Dragović G. Fremdsprachenunterricht (ent-)dramatisieren: Eine empirische Untersuchung zur Effizienz des dramapädagogischen Ansatzes im schulischen DaF-Unterricht mit speziellem Fokus auf Grammatik, [http://doc.rero.ch/], (Zugriff am 29.9.2019).

Even 2003: Even S. Drama Grammatik. Dramapädagogische Ansätze für den Grammatik-unterricht Deutsch als Fremdsprache. München: Iudicium.

Jelic 2011: Jelic V. Das Theater mit dem Englischbuch. Systematische Förderung mündlicher Kompetenzen mit dramapädagogischen Lehrverfahren, [http://publications.rwth-aachen.de/], (Zugriff am 29.9.2019).

Jin et al. 2012: Jin, F. et al. Prima A1. Deutsch für Jugendliche. Band 1. Schülerbuch. Berlin: Cornelsen Verlag.

Kao 1994: Kao S.-M. Classroom Interaction in a Drama-Oriented English Conversation Class of First-Year College Students in Taiwan: A Teacher-Researcher Study, [https://etd.ohiolink.edu/], (Zugriff am 10.10.2019).

Schewe 2000: Schewe M. DaF Stunden dramapädagogisch gestalten - wie mache ich das?, in: G. Schlemminger, T. Brysch, M. Schewe (Hrsg.), Pädagogische Konzepte für einen ganzheitlichen DaF-Unterricht, Berlin: Cornelsen Verlag, 72-105.

Tschurtschenthaler 2013: Tschurtschenthaler H. Drama-based foreign language learning. Münster: Waxmann.

Tselikas 1999: Tselikas E. Dramapädagogik im Sprachunterricht. Zürich: Orell Füssli. 


\title{
Georgina Dragović
}

\section{ŠKOLSKA NASTAVA NEMAČKOG JEZIKA KAO STRANOG SA ELEMENTIMA I BEZ ELEMENATA DRAMSKE PEDAGOGIJE: SA FOKUSOM NA UČENJE I PODUČAVANJE VOKABULARA}

\begin{abstract}
Rezime
Ovaj članak predstavlja kratak pregled autorkinog doktorskog rada koji se bavi istraživanjem razlika između dvaju pristupa u nastavi nemačkog jezika kao stranog u pogledu na kognitivne i afektivne varijable: između tradicionalnog i dramski orijentisanog pristupa nastavi. Istraživanje je sprovedeno u dve osnovne škole u Srbiji u okviru kog su učenici petog razreda $(n=60)$ iz tri odeljenja testirani i ispitivani putem raznih instrumenata. Podaci su analizirani deskriptivnom i inferencijalnom statistikom.

Dok je u doktorskom radu stavljen fokus na predavanje gramatike, ovaj rad ima za cilj da predstavi obradu novog vokabulara kao i njegovo uvežbavanje prema jednom i drugom pristupu nastavi. Teorijska rasprava dopunjena je pripremama za nastavu koje su korišćene tokom autorkine intervencije u kontrolnim odeljenjima, koja su učila tradicionalno, i eksperimentalnom odeljenju, koje je učilo po principima dramske pedagogije. Na primeru nastavne jedinice „Školski predmeti“ prikazano je kako se istom gradivu može pristupiti na dva različita načina. Kraj ovog rada čini sažetak rezultata istraživanja i zaključka koji se izvodi za rad u školskoj nastavi nemačkog jezika kao stranog.
\end{abstract}

Ključne reči: školski kontekst, dramska pedagogija, nemački jezik kao strani, poučavanje i učenje vokabulara. 